\title{
Characterizing Occupational Low Back Pain among Surgeons Working in Ministry of Health Hospitals in Jeddah City: Prevalence, Clinical Features, Risk, and Protective Factors
}

\author{
Khaled S. Aseri ${ }^{1}$, CMAB, Abeer A. Mulla² ${ }^{2}$ MBBS, Raghda M. Alwaraq ${ }^{3}$, MBBS, \\ Raneen J. Bahannan' ${ }^{2}$, MBBS \\ ${ }^{1}$ King Abdulaziz Medical City, Jeddah, King Saud bin Abdulaziz University for Health Sciences, Medical College \\ Department of Family and Community Medicine, Jeddah, Saudi Arabia \\ ${ }^{2}$ Faculty of Medicine, Ibn Sina National College for Medical Studies, Jeddah, Saudi Arabia \\ ${ }^{3}$ Faculty of Medicine, King Abdulaziz University, Jeddah, Saudi Arabia
}

\section{Correspondence}

Dr. Khaled S. Aseri

Department of Family and Community

Medicine, King Saud bin Abdulaziz University for Health Sciences, King Abdulaziz Medical City

P.O. Box 9515, Jeddah 21423

Saudi Arabia

e-M: kaserisaeed@gmail.com

Submission: 03 Nov. 2019

Accepted: 29 Dec. 2019

\section{Citation}

Aseri KS, Mulla AA, Alwaraq RM, Bahannan RJ. Characterizing occupational low back pain among surgeons working in Ministry of Health hospitals in Jeddah city: prevalence, clinical features and risk and protective factors. JKAU Med Sci 2019; 26 (2): 19-34. DOI: 10.4197/ Med.26-2.3

Copyright: $\odot$ The Author(s), YEAR. Publisher. The Journal of King Abdualziz University - Medical Sciences is an Official Publication of "King Abdulaziz University". It is an open-access article distributed under the terms of the Creative Commons Attribution Non-Commercial License, which permit unrestricted non-commercial use, distribution, and reproduction in any medium, provided the original work is properly cited.

\begin{abstract}
A cross-sectional study was conducted to investigate the prevalence as well as the determinants and clinical features of occupational low back pain among surgeons working at Ministry of Health hospitals in Jeddah, Saudi Arabia by exploring its history combined with time of onset after starting the operating room work and self-appraisal of the relationship with the surgical work. Factors and predictors such as professional, socio-demographic and lifestyle factors, medical history, etc. were collected and analyzed. Two hundred and sixteen surgeons (69.9\% males, mean age 39.03 years, median work: 5.00 years) responded. Prevalence of occupational low back pain was $55.8 \%$ (95\% Cl: 49.0\%, $62.3 \%$ ); high rates of inadequate management and self-treatment had a notable impact on all 4 domains. Multivariate regression showed two independent protective factors (regular physical exercise $[\mathrm{OR}=0.27, \mathrm{P}=0.002]$ and back health education [OR $=0.41, P=0.031]$ and two independent risk factors (high exposure to risky activities [OR $=1.06, \mathrm{P}=0.048]$ and presence of chronic pain other than back pain $[\mathrm{OR}=2.59, \mathrm{P}=0.012])$. More than one in two surgeons are likely to suffer from occupational low back pain. The protective roles of regular physical activity and back health education are highlighted.
\end{abstract}

\section{Keywords}

Occupational; Low back pain; Surgeons; Prevalence; Impact; Risk

Factors; Prevention; Education 


\section{Introduction}

$T$ he worldwide prevalence of chronic low back pain (LBP) was estimated between $4.2 \%$ and $25.4 \%$ depending on the population characteristics, region and age category ${ }^{[1]}$; with up to $42 \%$ reported point and lifetime prevalence ${ }^{[2,3]}$. Besides these disturbing epidemiological pictures, LBP has a major effect on social and professional activities since the functional capacity of the individual is restricted by pain; thus, it represents a significant cause of absenteeism ${ }^{[4]}$.

Focusing on the healthcare workers, LBP is an annoying problem that could affect the work process. In a recent prospective study conducted in France, approximately one-quarter of the healthcare workers with LBP did not return to their regular work during a 2-year follow-up period ${ }^{[5]}$. Estimates show that $47 \%-70 \%$ of healthcare workers had LBP, with a point-prevalence of $30 \%{ }^{[6,7]}$. In Kingdom of Saudi Arabia (KSA), a recent study conducted in the Eastern Region concluded that the majority of Saudi healthcare workers complain of LBP once in their lives with a prevalence of $79 \%$; most were work-related ${ }^{[8]}$.

On the other hand, approximately 4 out of 10 cases of LBP are attributed to occupational exposures ${ }^{[0]}$. Several work activities and professional circumstances have been identified as precursors for LBP, which makes occupational LBP (OLBP) a distinct entity.

Among the community of healthcare workers, surgeons are particularly vulnerable to experiencing OLBP compared to other specialties ${ }^{[6]}$. Studies showed that $70 \%-85 \%$ of surgeons complained from LBP in their lifetime ${ }^{[10,11]}$. Furthermore, chronic LBP among surgeons would eventually lead to significant impairments in work performance, which may affect the quality of patient's care and surgical outcomes and induce considerable healthcare costs, besides the substantial impact on the surgeon's quality of life ${ }^{[12]}$.

However, several studies conducted among surgeons did not explore the occupational nature of LBP, which may lead to misinterpretations of the presented epidemiological pictures and confusions with non-occupational LBP that may be pre-existing or due to extra-occupational etiology such as trauma, sport injuries, etc. Thus, identifying OLBP among surgeons would provide more accurate evaluation of the back-health risks related to the surgical profession, which would enable establishing more specific correlations with the work environment risk factors and protective measures.

The authors wanted to investigate the prevalence and clinical characteristics of OLBP among surgeons working in healthcare institutions of the Ministry of Health $(\mathrm{MOH})$ in Jeddah, Saudi Arabia; as well as to identify eventual independent risk or protective factors of an occupational or extra-occupational nature.

\section{Methods}

\section{Population and Setting}

This cross-sectional study involved surgeons working at five $\mathrm{MOH}$ hospitals in Jeddah, Saudi Arabia, including King Fahad General Hospital, King Abdul-Aziz Hospital and Oncology Center, Al Thagher Hospital, King Abdullah Medical Complex, and East Jeddah Hospital. Inclusion criteria applied for both gender and all nationality and age category residents, board-certified specialists and consultants in surgery. All surgical subspecialties were included, while other operatory room staff was not included. This study was approved by the Directorate of Health Affairs, $\mathrm{MOH}$, Jeddah; and researchers received relevant authorizations from all participating hospitals' directors and surgery departments' heads to collect the data specified in the study protocol.

\section{Sampling}

Sample size was calculated to detect $70 \%$ prevalence of low back pain ${ }^{[10]}$ among a finite population of 503 surgeons working in the participating centers, with a $95 \%$ confidence interval, $80 \%$ statistical power, and $5 \%$ type I error. The calculated sample size was $\mathrm{N}=197$.

A convenience sampling technique was used to include all eligible participants until reaching the target sample size, with respect of a proportional allocation by hospital as per the respective number of surgeons.

\section{Data Collection Tool}

This study used a semi-structured questionnaire that was designed by the authors, comprising a modified version of a validated questionnaire published in a previous study by Bin Homaid et al. ${ }^{[10]}$ that aimed to explore operatory room risk activities (ORRA) among operatory room staff in Makkah, Saudi Arabia. The 
questionnaire included the following nine parts: 1) Professional data: hospital name, subspecialty, years of experience, and work years; 2) Socio-demographic data: age, gender, marital status, etc.; 3) Lifestyle factors: smoking, physical exercise, hobbies, etc.; 4) Past medical history: specific back history (back trauma, surgery, injury, etc.), other medical and surgical history (other surgery, other trauma, co-morbidities, etc.); 5) Physical and psychological stress at work: perceived level of stress, hours of standing, hours of sitting, etc.; 6) ORRA, which explored surgeons' exposure to 8 different activities/postures (e.g. lifting, transferring, or pulling patients/objects) during a routine work time, as adapted from study by Bin Homaid et al. ${ }^{[10]}$ and that was modified by using a 5-point Likert type frequency scale (from never $=0$, to very frequently $=4$ ); 7) OLBP assessment, which was carried out by exploring history of LBP in addition to 2 discriminative criteria including time of onset (before vs after starting the operating room job) and self-appraisal of the relationship of LBP with the surgical work; 8) OLBP characteristics: pain severity, frequency and evolution pattern over the last 12 months, care-seeking behavior, etiology and diagnosis, and management; 9) Self-reported impact of OLBP on 4 dimensions including professional achievement, social relationships, psychological wellbeing, and activities of daily life.

After face- and content-validation of the questionnaire, an electronic version was adapted and published in an online survey platform and the related Uniform Resource Locator (URL) was obtained.

\section{Data Collection Method}

First, the online questionnaire URL was diffused via group messaging, by adding all phone numbers of all surgeons; these were provided beforehand by the hospitals' human resource administrators upon authorization of the respective directors and head managers. The questionnaire URL was accompanied with a short message soliciting for participation in the study and presenting its aim and relevance. However, due to the very low response rate (5.2\%), the researchers called each surgeon individually and reiterated the call for participation by presenting the importance of the study in a persuasive and constructive debate. The study URL was closed on 14 March 2019 after reaching 216 participants.

\section{Statistical methods}

Statistical analysis was performed with the IBM SPSS Statistics for Windows, Version 21 (IBM Corp., Armonk, NY USA). The prevalence of OLBP was calculated as the percentage of surgeons who declared having LBP with onset after start of operatory room work (to exclude anterior symptoms) and being fairly associated to the profession as per the participant's subjective judgment on a Likert-type probability scale (to exclude extraoccupational etiology). Results are presented with a 95\% confidence interval. Descriptive statistics were used to present socio-demographic, professional, lifestyle factors and job-associated stressors, in addition to OLBP characteristics and multidimensional impact. Categorical variables were presented as frequency and percentage, while numerical variables were presented as mean \pm Standard Deviation (SD) for normally distributed variables and median (centile) for non-normally distributed ones. Regarding risky activities, an ORRA score (ORRAS) was calculated as the sum of the eight items' scores to indicate the overall level of exposure (ORRAS range $=0,32$ ). The internal consistency of related parts of the questionnaire was assessed by calculating Cronbach's alpha. Analysis of factors associated with OLBP used chi-squared test or Fisher's exact test for categorical variables, as appropriate, independent t-test for normally distributed and Mann-Whitney $U$ test for non-normally distributed ones. Multivariate binary logistic regression was carried out to analyze independent risk factors of OLBP by including all statistically significant factors; results were presented as odds-ratio (OR) with 95\% confidence interval $(95 \% \mathrm{Cl})$. A $p$ value of $<0.05$ was considered to reject the null hypothesis.

\section{Results}

\section{Participants' Demographic and Professional Characteristics}

Two hundred and sixteen surgeons responded to the questionnaire, $69.9 \%$ males, mean (SD) age of 39.03 (9.82) years and $78.3 \%$ were married. Of the participants, $32.7 \%$ were residents, $33.2 \%$ boardcertified specialists, and $34.1 \%$ were consultants, with median (P90) experience of 10.00 (25.00) years; with sub Table 1). 
Table 1. Participants' demographic and professional characteristics.

\begin{tabular}{|c|c|c|c|}
\hline Parameter & Category & Frequency & Percentage \\
\hline \multicolumn{4}{|l|}{ Socio-demographic data } \\
\hline \multirow[t]{2}{*}{ Gender } & Male & 158 & $69.9 \%$ \\
\hline & Female & 68 & $30.1 \%$ \\
\hline Age (Years) & Mean, SD & 39.03 & $9.82 \%$ \\
\hline \multirow[t]{4}{*}{ Marital Status } & Single & 42 & $18.6 \%$ \\
\hline & Married & 177 & $78.3 \%$ \\
\hline & Divorced & 5 & $2.2 \%$ \\
\hline & Widowed & 2 & $0.9 \%$ \\
\hline \multirow[t]{3}{*}{ No. children } & None & 59 & $26.1 \%$ \\
\hline & $1-2$ & 69 & $30.5 \%$ \\
\hline & $3+$ & 80 & $35.4 \%$ \\
\hline \multicolumn{4}{|l|}{ Professional data } \\
\hline \multirow[t]{5}{*}{ Hospital } & Al Thagher Hospital & 25 & $11.1 \%$ \\
\hline & East Jeddah Hospital & 42 & $18.6 \%$ \\
\hline & King Abdul-Aziz Hospital and Oncology Center & 64 & $28.3 \%$ \\
\hline & King Abdullah Medical Complex & 28 & $12.4 \%$ \\
\hline & King Fahad General Hospital & 67 & $29.6 \%$ \\
\hline \multirow[t]{3}{*}{ Degree } & Consultant & 77 & $34.1 \%$ \\
\hline & Resident & 74 & $32.7 \%$ \\
\hline & Board-certified specialist & 75 & $33.2 \%$ \\
\hline Years of experience & Median, P90 & 10.00 & 25.00 \\
\hline \multirow{14}{*}{ Subspecialty } & Bariatric & 6 & $2.7 \%$ \\
\hline & Breast Oncology/Surgery & 5 & $2.2 \%$ \\
\hline & General Surgery & 81 & $35.8 \%$ \\
\hline & Orthopedic & 25 & $11.1 \%$ \\
\hline & Obstetrics/Gynecology & 18 & $8.0 \%$ \\
\hline & Otolaryngology & 14 & $6.2 \%$ \\
\hline & Pediatric Surgery & 3 & $1.3 \%$ \\
\hline & Neurosurgery & 5 & $2.2 \%$ \\
\hline & Urology & 22 & $9.7 \%$ \\
\hline & Maxillofacial & 17 & $7.5 \%$ \\
\hline & Plastic Surgery & 8 & $3.5 \%$ \\
\hline & Surgical Oncology & 2 & $0.9 \%$ \\
\hline & Gynecological Oncology & 7 & $3.1 \%$ \\
\hline & Other (Specified) & 13 & $5.8 \%$ \\
\hline Years of work & Median, P90 & 5.00 & 20.00 \\
\hline
\end{tabular}

Because of missing data, all frequencies do not sum up to the total. Values are frequency/percentage, except if otherwise specified. P90: $90^{\text {th }}$ centile; other sub-specialties included: ophthalmology (3), thoracic surgery (3), vascular surgery (2), colorectal surgery (2), anesthesia (1), gastroenterology endoscopic surgery (1), and gynecology and infertility (1).

\section{Participants' Lifestyle and Clinical Characteristics}

Of the total participants, active smoking was present among $28.3 \%$ with a median (P90) duration of 9.0 (20.0) years of smoking and an average of 5 (20) cigarettes per day. Other lifestyle characteristics showed low rates of regular exercise (36.3\%) and hobbies and extraprofessional activities (21.7\%). Back history showed $16.8 \%$ of back injury in various circumstances including trauma $(8.0 \%)$, sport injury $(4.0)$, surgery $(1.8 \%)$, or other causes $(8.8 \%)$. Other medical data were characterized by high prevalence of overweight (35.8\%) and obesity
(36.3\%), with remarkably low medical history of hypertension (12.4\%) and type II diabetes (5.3\%) (Table 2).

\section{Physical and Psychological Stress at Work}

A majority of the surgeons declared undergoing high $(40.7 \%)$ or very high $(15.9 \%)$ levels of stress during their work. The median (P90) average standing and sitting hours in a workday were $9.00(20.00)$ and 5.00 (20.00) hours, respectively. Regarding operatory room risky activities (ORRA), the most frequently performed activities were lifting objects above the 
Characterizing Occupational Low Back Pain among Surgeons Working in Ministry of Health Hospitals in Jeddah City... K.S. Aseri et al.

Table 2. Participants' lifestyle and clinical characteristics

\begin{tabular}{|c|c|c|c|}
\hline Parameter & Category & Frequency & Percent \\
\hline \multicolumn{4}{|l|}{ Lifestyle data } \\
\hline \multirow[t]{4}{*}{ Home-job transportation mean } & Drive own car & 147 & $65.0 \%$ \\
\hline & Private driver & 52 & $23.0 \%$ \\
\hline & By taxi & 8 & $3.5 \%$ \\
\hline & On foot & 19 & $8.4 \%$ \\
\hline \multirow[t]{4}{*}{ Smoking } & Never & 134 & $59.3 \%$ \\
\hline & Ever & 92 & $40.7 \%$ \\
\hline & Previously & 27 & $11.9 \%$ \\
\hline & Currently & 64 & $28.3 \%$ \\
\hline \multirow[t]{2}{*}{ Type } & Cigarettes & 55 & $24.3 \%$ \\
\hline & Shisha & 36 & $15.9 \%$ \\
\hline Smoking duration & Median, P90 & 9.00 & 20.00 \\
\hline No. cigarettes per day & Median, P90 & 5.00 & 20.00 \\
\hline \multirow[t]{2}{*}{ Regular physical exercise } & No & 75 & $33.2 \%$ \\
\hline & Yes & 151 & $66.8 \%$ \\
\hline Weekly exercise frequency & Median, SD & 3.00 & 6.00 \\
\hline \multirow[t]{3}{*}{ Exercise (3 categories) } & None & 75 & $33.2 \%$ \\
\hline & Irregular ( $<3$ times/week) & 69 & $30.5 \%$ \\
\hline & Regular ( $\geq 3$ times/week) & 82 & $36.3 \%$ \\
\hline \multirow[t]{2}{*}{ Hobbies and extra-professional activities } & No & 177 & $78.3 \%$ \\
\hline & Yes & 49 & $21.7 \%$ \\
\hline \multirow[t]{4}{*}{ Holidays and travel } & Always & 101 & $44.7 \%$ \\
\hline & Often & 92 & $40.7 \%$ \\
\hline & Rarely & 30 & $13.3 \%$ \\
\hline & Never & 3 & $1.3 \%$ \\
\hline \multicolumn{4}{|l|}{ Medical data } \\
\hline Height (cm) & Mean, SD & 169.80 & 9.42 \\
\hline Weight (kg) & Mean, SD & 83.25 & 20.23 \\
\hline BMI $\left(\mathrm{kg} / \mathrm{m}^{2}\right)$ & Mean, SD & 28.75 & 5.91 \\
\hline \multirow[t]{6}{*}{ BMI categories $\left(\mathrm{kg} / \mathrm{m}^{2}\right)$} & Underweight $(<18.5)$ & 1 & $0.4 \%$ \\
\hline & Normal $(18.5-<25)$ & 62 & $27.4 \%$ \\
\hline & Overweight $(25-<30)$ & 81 & $35.8 \%$ \\
\hline & Class I obesity $(30-<35)$ & 51 & $22.6 \%$ \\
\hline & Class II obesity $(35-<40)$ & 20 & $8.8 \%$ \\
\hline & Class III obesity (40+) & 11 & $4.9 \%$ \\
\hline \multirow[t]{3}{*}{ Current pregnancy } & No & 64 & $28.3 \%$ \\
\hline & Yes & 4 & $1.8 \%$ \\
\hline & N/A (males) & 158 & $69.9 \%$ \\
\hline \multirow[t]{6}{*}{ History of back injury } & Never & 188 & $83.2 \%$ \\
\hline & Ever $^{\S}$ & 38 & $16.8 \%$ \\
\hline & Trauma & 18 & $8.0 \%$ \\
\hline & Sport injury & 9 & $4.0 \%$ \\
\hline & Surgery & 4 & $1.8 \%$ \\
\hline & Other & 20 & $8.8 \%$ \\
\hline \multirow[t]{2}{*}{ Other surgical history } & No & 151 & $66.8 \%$ \\
\hline & Yes & 75 & $33.2 \%$ \\
\hline \multirow[t]{2}{*}{ Other trauma history } & No & 175 & $77.4 \%$ \\
\hline & Yes & 51 & $22.6 \%$ \\
\hline \multirow[t]{6}{*}{ Other medical history } & No & 156 & $69.0 \%$ \\
\hline & Yes & 70 & $31.0 \%$ \\
\hline & Hypertension & 28 & $12.4 \%$ \\
\hline & Type ll diabetes & 12 & $5.3 \%$ \\
\hline & B.A or allergic rhinitis & 13 & $5.8 \%$ \\
\hline & Other & 24 & $10.6 \%$ \\
\hline
\end{tabular}

Because of missing data, all frequencies do not sum up to the total. Values are frequency/percentage, except if otherwise specified. P90: $90^{\text {th }}$ centile; ${ }^{\S}$ a participant may have more than one injury; other medical history included hypothyroidism (7 cases), coronary artery disease (2), hyperlipidemia (3), hyperprolactinemia (2), migraine (2), anemia (1), atrial fibrillation (1), gout (1), hyperthyroidism (1), rheumatological disease (1), simple renal cyst (1), and thrombophilia A (1), in addition to two unspecified cases. 
Characterizing Occupational Low Back Pain among Surgeons Working in Ministry of Health Hospitals in Jeddah City... K.S. Aseri et al.

Table 3. Assessment of physical and psychological stress at work among surgeons

\begin{tabular}{|c|c|c|c|}
\hline Parameter & Level & Frequency & Percent \\
\hline \multirow[t]{4}{*}{ Stress level at work } & Low & 10 & $4.4 \%$ \\
\hline & Moderate & 88 & $38.9 \%$ \\
\hline & High & 92 & $40.7 \%$ \\
\hline & Very high & 36 & $15.9 \%$ \\
\hline Average standing hours per workday & Median, P90 & 9.00 & 20.00 \\
\hline Average sitting hours per workday & Median, P90 & 5.00 & 20.00 \\
\hline \multicolumn{4}{|l|}{ ORRA } \\
\hline \multirow[t]{5}{*}{ Lifting objects above the waist } & Never & 22 & $9.7 \%$ \\
\hline & Rarely & 63 & $27.9 \%$ \\
\hline & Occasionally & 72 & $31.9 \%$ \\
\hline & Frequently & 57 & $25.2 \%$ \\
\hline & Very frequently & 12 & $5.3 \%$ \\
\hline \multirow[t]{5}{*}{ Rotating torso while bearing weight } & Never & 58 & $25.7 \%$ \\
\hline & Rarely & 75 & $33.2 \%$ \\
\hline & Occasionally & 60 & $26.5 \%$ \\
\hline & Frequently & 27 & $11.9 \%$ \\
\hline & Very frequently & 6 & $2.7 \%$ \\
\hline \multirow[t]{5}{*}{ Bending to lift an item from floor level } & Never & 40 & $17.7 \%$ \\
\hline & Rarely & 67 & $29.6 \%$ \\
\hline & Occasionally & 60 & $26.5 \%$ \\
\hline & Frequently & 39 & $17.3 \%$ \\
\hline & Very frequently & 20 & $8.8 \%$ \\
\hline \multirow[t]{5}{*}{ Transferring a patient from bed to chair/chair to bed } & Never & 42 & $18.6 \%$ \\
\hline & Rarely & 70 & $31.0 \%$ \\
\hline & Occasionally & 54 & $23.9 \%$ \\
\hline & Frequently & 50 & $22.1 \%$ \\
\hline & Very frequently & 10 & $4.4 \%$ \\
\hline \multirow[t]{5}{*}{ Transferring a patient onto a stretcher } & Never & 50 & $22.1 \%$ \\
\hline & Rarely & 67 & $29.6 \%$ \\
\hline & Occasionally & 47 & $20.8 \%$ \\
\hline & Frequently & 51 & $22.6 \%$ \\
\hline & Very frequently & 11 & $4.9 \%$ \\
\hline \multirow[t]{5}{*}{ Ambulating a patient } & Never & 72 & $31.9 \%$ \\
\hline & Rarely & 87 & $38.5 \%$ \\
\hline & Occasionally & 45 & $19.9 \%$ \\
\hline & Frequently & 19 & $8.4 \%$ \\
\hline & Very frequently & 3 & $1.3 \%$ \\
\hline \multirow[t]{5}{*}{ Pulling a patient up the bed } & Never & 41 & $18.1 \%$ \\
\hline & Rarely & 96 & $42.5 \%$ \\
\hline & Occasionally & 52 & $23.0 \%$ \\
\hline & Frequently & 30 & $13.3 \%$ \\
\hline & Very frequently & 7 & $3.1 \%$ \\
\hline \multirow[t]{5}{*}{ Repositioning a patient in bed } & Never & 35 & $15.5 \%$ \\
\hline & Rarely & 91 & $40.3 \%$ \\
\hline & Occasionally & 62 & $27.4 \%$ \\
\hline & Frequently & 29 & $12.8 \%$ \\
\hline & Very frequently & 9 & $4.0 \%$ \\
\hline \multirow{3}{*}{$\begin{array}{l}\text { Education/ information received on how to protect back and } \\
\text { prevent LBP? }\end{array}$} & No & 125 & $55.3 \%$ \\
\hline & Yes, but inadequate & 55 & $24.3 \%$ \\
\hline & Yes, adequate & 46 & $20.4 \%$ \\
\hline
\end{tabular}

Because of missing data, all frequencies do not sum up to the total. Values are frequency/percentage, except if otherwise specified. P90: $90^{\text {th }}$ centile; OR: operating room risky activities; LBP: low back pain; operatory room risk activities levels: Never, Rarely: 1-2 times per month, Occasionally: 1-2 times per week, Frequently: several times per week, Very frequently: daily 
waist, transferring a patient from bed to chair/chair to bed and transferring a patient onto a stretcher. Further, only $44.7 \%$ reported having received information/ education on how to protect their back and prevent LBP and only $20.4 \%$ deemed the education received as adequate (Table 3 ).

Analysis of ORRAS showed mean $=12, \mathrm{SD}=6.30$ and median $=12$, and study of the distribution showed Kolmogorov-Smirnov test (statistics $=0.083, p=0.001$ ) and Shapiro-Wilk test (statistics $=0.984, p=0.012$ ), indicating that the variable is not normally distributed. Internal consistency analysis of the ORRA scale including the eight items showed Cronbach's alpha = 0.868 , indicating a high reliability of the subscale.

\section{Characterization of OLBP}

History of LBP was reported in 192 of the total surgeons, indicating a prevalence of $85.0 \%(95 \% \mathrm{Cl}=79.6 \%$, $89.4 \%$ ). Of the 192 surgeons, 156 surgeons $(69.0 \%$ of the total surgeons) declared that their LBP first occurred after starting their OR activity and $150(66.4 \%)$ believed it is probably, very probably or definitely related to their job; which results in 126 having both criteria and more likelihood of having OLBP (prevalence $[95 \% \mathrm{Cl}]=$ $55.8 \%[49.0 \%, 62.3 \%])$.

Findings of clinical characteristics of OLBP are depicted in Table 4 . These revealed that $32.5 \%$ of the afflicted surgeons consulted a physician for their OLBP and $25.4 \%$ underwent an MRI or CT-scan; and the proposed etiological diagnoses were muscular injury or dysfunction (20.6\%), discopathy (14.3\%), degenerative disease $(4.0 \%)$, sacroiliitis (1.6\%) and functional LBP without any anatomical lesion (4.8\%); whereas $54.8 \%$ of the cases remained without diagnosis. Yearly frequency of OLBP episodes ranged between 0 to $300+$ episodes per year (median $=6, \mathrm{P} 90=33$ ), with the latest episode occurring in the past few days among $38.1 \%$ of the cases. Severity of the pain showed mean (SD) pain score $=5.01$ (1.84) for the latest episode and 6.45 (2.18) for the most painful episode. Regarding management, rest (48.4\%), NSAIDs (27.6\%) and physiotherapy (26.6\%) were the most frequently used options; however, opioid analgesics have been used by $2.6 \%$ of the afflicted surgeons (Fig. 1). Regarding the impact on life domains, OLBP yielded great impact on professional achievement, social relationships, ADLs and psychological well-being in $13.5 \%, 5.6 \%$, $12.7 \%$ and $24.6 \%$ of the cases, respectively; while a moderate impact was reported among $33.3 \%$ to $40.5 \%$ depending on the domain (Table 4).

\section{Factors Associated with OLBP}

Demographic and professional factors associated with OLBP as well as lifestyle and clinical factors are depicted in Table 5. No statistically significant association of OLBP was found with any of the investigated demographic

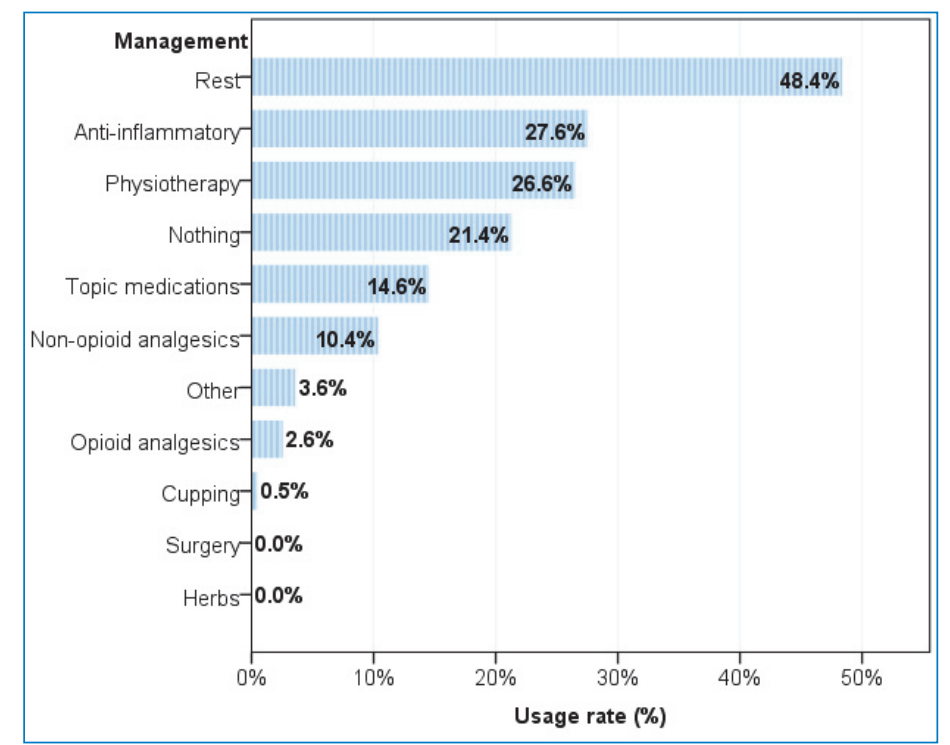

Figure 1. Management options used among surgeons who declared ever experiencing low back pain $(\mathrm{N}=192)$ Bars represent the percentage of surgeons of who already used the given management option among those who declared having ever had low back pain. A participant may use more than one option. Other management options included massage and physiotherapy (5 cases), stretching or exercise (4), weight reduction (1) and behavioral change (1) 
Characterizing Occupational Low Back Pain among Surgeons Working in Ministry of Health Hospitals in Jeddah City... K.S. Aseri et al.

Table 4. Characteristics and impact of occupational low back pain among afflicted surgeons $(\mathrm{N}=126)$

\begin{tabular}{|c|c|c|c|}
\hline Parameter & Category & Frequency & Percent \\
\hline \multirow[t]{2}{*}{ Consulted a physician } & No & 85 & $67.5 \%$ \\
\hline & Yes & 41 & $32.5 \%$ \\
\hline \multirow[t]{2}{*}{ Had MRI and or CT-scan } & No & 94 & $74.6 \%$ \\
\hline & Yes & 32 & $25.4 \%$ \\
\hline \multirow[t]{6}{*}{ Diagnosis / etiology } & Discopathy & 18 & $14.3 \%$ \\
\hline & Muscular & 26 & $20.6 \%$ \\
\hline & Degenerative & 5 & $4.0 \%$ \\
\hline & Sacroiliitis & 2 & $1.6 \%$ \\
\hline & Non-specific / Functional & 6 & $4.8 \%$ \\
\hline & Unknown / Not Specified & 69 & $54.8 \%$ \\
\hline \multirow[t]{6}{*}{ In your opinion, is your LBP related to your job? } & No not related & 0 & $0.0 \%$ \\
\hline & Probably not & 0 & $0.0 \%$ \\
\hline & Possibly & 0 & $0.0 \%$ \\
\hline & Probably & 36 & $28.6 \%$ \\
\hline & Very probably & 31 & $24.6 \%$ \\
\hline & Definitely & 59 & $46.8 \%$ \\
\hline No. episodes past year & Median, P90 & 6 & 33 \\
\hline \multirow[t]{4}{*}{ Latest episode time } & $>3$ months & 21 & $16.7 \%$ \\
\hline & $1-3$ months & 21 & $16.7 \%$ \\
\hline & 1 week - 1 month & 36 & $28.6 \%$ \\
\hline & A few days ago & 48 & $38.1 \%$ \\
\hline \multirow[t]{4}{*}{ Evolution pattern } & Decreasing & 31 & $24.6 \%$ \\
\hline & By intermittent peaks & 60 & $47.6 \%$ \\
\hline & Constant intensity & 27 & $21.4 \%$ \\
\hline & Increasing & 8 & $6.3 \%$ \\
\hline Latest episode severity & Mean, SD & 5.01 & 1.84 \\
\hline Most painful episode severity & Mean, SD & 6.45 & 2.18 \\
\hline \multirow[t]{5}{*}{ Usual Management } & Nothing (wait until it passes away) & 14 & $11.1 \%$ \\
\hline & Rest & 57 & $45.2 \%$ \\
\hline & Self-medication & 46 & $36.5 \%$ \\
\hline & I see a physician/specialist & 3 & $2.4 \%$ \\
\hline & Other, specify & 6 & $4.8 \%$ \\
\hline \multirow[t]{2}{*}{ Chronic pain in other area } & No & 70 & $55.6 \%$ \\
\hline & Yes & 56 & $44.4 \%$ \\
\hline \multicolumn{4}{|l|}{ Impact of $\angle B P$} \\
\hline \multirow[t]{4}{*}{ Job achievement } & Not at all & 13 & $10.3 \%$ \\
\hline & Very little & 46 & $36.5 \%$ \\
\hline & Somewhat & 50 & $39.7 \%$ \\
\hline & To a great extent & 17 & $13.5 \%$ \\
\hline \multirow[t]{4}{*}{ Social relationships } & Not at all & 23 & $18.3 \%$ \\
\hline & Very little & 45 & $35.7 \%$ \\
\hline & Somewhat & 51 & $40.5 \%$ \\
\hline & To a great extent & 7 & $5.6 \%$ \\
\hline \multirow[t]{4}{*}{ Activities of daily life } & Not at all & 17 & $13.5 \%$ \\
\hline & Very little & 42 & $33.3 \%$ \\
\hline & Somewhat & 51 & $40.5 \%$ \\
\hline & To a great extent & 16 & $12.7 \%$ \\
\hline \multirow[t]{4}{*}{ Psychological wellbeing } & Not at all & 11 & $8.7 \%$ \\
\hline & Very little & 42 & $33.3 \%$ \\
\hline & Somewhat & 42 & $33.3 \%$ \\
\hline & To a great extent & 31 & $24.6 \%$ \\
\hline
\end{tabular}

Values are frequency/percentage, except if otherwise specified. P90: $90^{\text {th }}$ centile; SD: Standard deviation; LBP: Low back pain; OLBP: Occupational low back pain; OLBP criteria: $1=L B P$ first occurred after starting the $O R$ job, $2=L B P$ described by the participant as being probably related to $j o b, 3=L B P$ first occurred after starting OR job and described by participant as being probably related 
Characterizing Occupational Low Back Pain among Surgeons Working in Ministry of Health Hospitals in Jeddah City... K.S. Aseri et al.

Table 5. Demographic, professional, lifestyle and clinical factors associated with occupational low back pain among surgeons

\begin{tabular}{|c|c|c|c|c|c|c|}
\hline \multirow{3}{*}{ Parameter } & \multirow{3}{*}{ Category } & \multicolumn{4}{|c|}{ OLBP } & \multirow{3}{*}{ P-value } \\
\hline & & \multicolumn{2}{|c|}{ No $(\mathrm{N}=100)$} & \multicolumn{2}{|c|}{ Yes $(N=126)$} & \\
\hline & & Frequency & Percent & Frequency & Percent & \\
\hline \multicolumn{7}{|l|}{ Socio-demographic factors } \\
\hline \multirow[t]{2}{*}{ Gender } & Male & 72 & $45.6 \%$ & 86 & $54.4 \%$ & 0 \\
\hline & Female & 28 & $41.2 \%$ & 40 & $58.8 \%$ & 0.542 \\
\hline Age (years) & Mean, SD & 39.24 & 10.54 & 38.86 & $9.25 \%$ & $0.772^{t}$ \\
\hline \multirow[t]{4}{*}{ Marital Status } & Single & 19 & $45.2 \%$ & 23 & $54.8 \%$ & 0 \\
\hline & Married & 78 & $44.1 \%$ & 99 & $55.9 \%$ & 0 \\
\hline & Divorced & 1 & $20.0 \%$ & 4 & $80.0 \%$ & 0 \\
\hline & Widowed & 2 & $100.0 \%$ & 0 & $0.0 \%$ & 0.292 \\
\hline \multirow[t]{3}{*}{ No. children } & None & 26 & $44.1 \%$ & 33 & $55.9 \%$ & 0 \\
\hline & $1-2$ & 32 & $46.4 \%$ & 37 & $53.6 \%$ & 0 \\
\hline & $3+$ & 35 & $43.8 \%$ & 45 & $56.3 \%$ & 0.943 \\
\hline \multicolumn{7}{|l|}{ Professional factors } \\
\hline \multirow[t]{3}{*}{ Degree } & Consultant & 36 & $46.8 \%$ & 41 & $53.2 \%$ & 0 \\
\hline & Resident & 36 & $48.6 \%$ & 38 & $51.4 \%$ & 0 \\
\hline & Board-certified specialist & 28 & $37.3 \%$ & 47 & $62.7 \%$ & 0.328 \\
\hline Years of experience & Median, P90 & 9.00 & $27.70 \%$ & 10.50 & $25.00 \%$ & $0.475^{\mathrm{M}}$ \\
\hline \multirow[t]{14}{*}{ Subspecialty } & Bariatric & 3 & $50.0 \%$ & 3 & $50.0 \%$ & 0 \\
\hline & Breast oncology/surgery & 3 & $60.0 \%$ & 2 & $40.0 \%$ & 0 \\
\hline & General surgery & 34 & $42.0 \%$ & 47 & $58.0 \%$ & 0 \\
\hline & Orthopedic & 11 & $44.0 \%$ & 14 & $56.0 \%$ & 0 \\
\hline & Ob-gyne & 5 & $27.8 \%$ & 13 & $72.2 \%$ & 0 \\
\hline & ENT & 6 & $42.9 \%$ & 8 & $57.1 \%$ & 0 \\
\hline & Pediatric surg. & 3 & $100.0 \%$ & 0 & $0.0 \%$ & 0 \\
\hline & Neurosurgery & 3 & $60.0 \%$ & 2 & $40.0 \%$ & 0 \\
\hline & Urology & 6 & $27.3 \%$ & 16 & $72.7 \%$ & 0 \\
\hline & Maxillofacial & 11 & $64.7 \%$ & 6 & $35.3 \%$ & 0 \\
\hline & Plastic surgery & 4 & $50.0 \%$ & 4 & $50.0 \%$ & 0 \\
\hline & Surgical oncologist & 1 & $50.0 \%$ & 1 & $50.0 \%$ & 0 \\
\hline & Gynecological oncology & 3 & $42.9 \%$ & 4 & $57.1 \%$ & 0 \\
\hline & Other (specified) & 7 & $53.8 \%$ & 6 & $46.2 \%$ & 0.440 \\
\hline Years of work & Median, P90 & 5.00 & $19.90 \%$ & 6.00 & $20.60 \%$ & $0.080^{\mathrm{M}}$ \\
\hline \multicolumn{7}{|l|}{ Lifestyle factors } \\
\hline \multirow[t]{4}{*}{ Home-job transportation mean } & Drive own car & 64 & $43.5 \%$ & 83 & $56.5 \%$ & 0 \\
\hline & Private driver & 21 & $40.4 \%$ & 31 & $59.6 \%$ & 0 \\
\hline & By taxi & 5 & $62.5 \%$ & 3 & $37.5 \%$ & 0 \\
\hline & Onfoot & 10 & $52.6 \%$ & 9 & $47.4 \%$ & 0.579 \\
\hline \multirow[t]{4}{*}{ Smoking } & Never & 61 & $45.5 \%$ & 73 & $54.5 \%$ & 0 \\
\hline & Ever: & 39 & $42.4 \%$ & 53 & $57.6 \%$ & 0.642 \\
\hline & Previously & 11 & $40.7 \%$ & 16 & $59.3 \%$ & 0 \\
\hline & Currently & 28 & $43.8 \%$ & 36 & $56.3 \%$ & 0.791 \\
\hline \multirow[t]{2}{*}{ Type } & Cigarettes & 24 & $43.6 \%$ & 31 & $56.4 \%$ & 0 \\
\hline & Shisha & 15 & $41.7 \%$ & 21 & $58.3 \%$ & 0.853 \\
\hline Smoking duration & Median, P90 & 10.00 & $18.80 \%$ & 9.00 & $20.0 \%$ & $0.967^{\mathrm{M}}$ \\
\hline No. cigarettes per day & Median, P90 & 10.00 & $20.0 \%$ & 5 & $20.0 \%$ & $0.641^{\mathrm{M}}$ \\
\hline \multirow[t]{2}{*}{ Regular physical exercise } & No & 23 & $30.7 \%$ & 52 & $69.3 \%$ & 0 \\
\hline & Yes & 77 & $51.0 \%$ & 74 & $49.0 \%$ & $0.004^{*}$ \\
\hline \multirow[t]{3}{*}{ Exercise (times/week) } & None & 23 & $30.7 \%$ & 52 & $69.3 \%$ & 0 \\
\hline & Irregular & 29 & $42.0 \%$ & 40 & $58.0 \%$ & 0 \\
\hline & Regular & 48 & $58.5 \%$ & 34 & $41.5 \%$ & $0.002^{*}$ \\
\hline
\end{tabular}


Characterizing Occupational Low Back Pain among Surgeons Working in Ministry of Health Hospitals in Jeddah City... K.S. Aseri et al.

Table 5. Demographic, professional, lifestyle and clinical factors associated with occupational low back pain among surgeons-(CONTINUED)

\begin{tabular}{|c|c|c|c|c|c|c|}
\hline \multirow{3}{*}{ Parameter } & \multirow{3}{*}{ Category } & \multicolumn{4}{|c|}{ OLBP } & \multirow{3}{*}{ P-value } \\
\hline & & \multicolumn{2}{|c|}{ No $(\mathrm{N}=100)$} & \multicolumn{2}{|c|}{ Yes $(\mathrm{N}=126)$} & \\
\hline & & Frequency & Percent & Frequency & Percent & \\
\hline \multirow{2}{*}{$\begin{array}{l}\text { Hobbies and extra-professional } \\
\text { activities }\end{array}$} & No & 76 & $42.9 \%$ & 101 & $57.1 \%$ & 0 \\
\hline & Yes & 24 & $49.0 \%$ & 25 & $51.0 \%$ & 0.451 \\
\hline \multirow[t]{4}{*}{ Holidays and travel } & Always & 43 & $42.6 \%$ & 58 & $57.3 \%$ & 0 \\
\hline & Often & 43 & $46.7 \%$ & 49 & $53.3 \%$ & 0 \\
\hline & Rarely & 14 & $46.7 \%$ & 16 & $53.3 \%$ & 0 \\
\hline & Never & 0 & $0.0 \%$ & 3 & $100.0 \%$ & 0.424 \\
\hline \multicolumn{7}{|l|}{ Clinical factor } \\
\hline Height $(\mathrm{cm})$ & Mean, SD & 169.51 & $9,99 \%$ & 170,03 & $8,98 \%$ & $0.680^{t}$ \\
\hline Weight (kg) & Mean, SD & 83.86 & $22.32 \%$ & 82.76 & $18.48 \%$ & $0.686^{t}$ \\
\hline BMI $\left(\mathrm{kg} / \mathrm{m}^{2}\right)$ & Mean, SD & 29.05 & $6.45 \%$ & 28.52 & $5.47 \%$ & $0.506^{t}$ \\
\hline \multirow[t]{2}{*}{ History of any back injury } & Never & 79 & $42.0 \%$ & 109 & $58.0 \%$ & 0 \\
\hline & Ever $^{\S}$ & 21 & $55.3 \%$ & 17 & $44.7 \%$ & 0.134 \\
\hline \multirow[t]{2}{*}{ Back trauma } & No & 88 & $42.3 \%$ & 120 & $57.7 \%$ & 0 \\
\hline & Yes & 12 & $66.7 \%$ & 6 & $33.3 \%$ & $0.046^{*}$ \\
\hline \multirow[t]{2}{*}{ Back sport injury } & No & 93 & $42.9 \%$ & 124 & $57.1 \%$ & 0 \\
\hline & Yes & 7 & $77.8 \%$ & 2 & $22.2 \%$ & $0.081^{\mathrm{F}}$ \\
\hline \multirow[t]{2}{*}{ Back surgery } & No & 99 & $44.6 \%$ & 123 & $55.4 \%$ & 0 \\
\hline & Yes & 1 & $25.0 \%$ & 3 & $75.0 \%$ & $0.632^{F}$ \\
\hline \multirow[t]{2}{*}{ Other back injury } & No & 91 & $44.2 \%$ & 115 & $55.8 \%$ & 0 \\
\hline & Yes & 9 & $45.0 \%$ & 11 & $55.0 \%$ & 0.943 \\
\hline \multirow[t]{2}{*}{ Other surgical history } & No & 66 & $43.7 \%$ & 85 & $56.3 \%$ & 0 \\
\hline & Yes & 34 & $45.3 \%$ & 41 & $54.7 \%$ & 0.817 \\
\hline \multirow[t]{2}{*}{ Other trauma history } & No & 84 & $48.0 \%$ & 91 & $52.0 \%$ & 0 \\
\hline & Yes & 16 & $31.4 \%$ & 35 & $68.6 \%$ & $0.035^{*}$ \\
\hline \multirow[t]{2}{*}{ Other chronic pain history } & No & 50 & $41.7 \%$ & 70 & $58.3 \%$ & 0 \\
\hline & Yes & 16 & $22.2 \%$ & 56 & $77.8 \%$ & $0.006^{*}$ \\
\hline \multirow[t]{2}{*}{ Other medical history } & No & 77 & $49.4 \%$ & 79 & $50.6 \%$ & 0 \\
\hline & Yes & 23 & $32.9 \%$ & 47 & $67.1 \%$ & $0.021^{*}$ \\
\hline \multicolumn{7}{|l|}{ Physical and psychological stress at work } \\
\hline \multirow[t]{4}{*}{ Perceived-stress level at work } & Low & 6 & $60.0 \%$ & 4 & $40.0 \%$ & 0 \\
\hline & Moderate & 43 & $48.9 \%$ & 45 & $51.1 \%$ & 0 \\
\hline & High & 40 & $43.5 \%$ & 52 & $56.5 \%$ & 0 \\
\hline & Very high & 11 & $30.6 \%$ & 25 & $69.4 \%$ & 0.210 \\
\hline Average standing hours per workday & Median, P90 & 5.00 & $8.0 \%$ & 6.00 & $8.0 \%$ & 0.057 \\
\hline Average sitting hours per workday & Median, P90 & 3.00 & $5.90 \%$ & 2.50 & $4.0 \%$ & 0.051 \\
\hline ORRAS & Mean, SD & 11.02 & $6.43 \%$ & 12.98 & $6.08 \%$ & $0.020^{* t}$ \\
\hline \multirow[t]{3}{*}{ Received education to prevent LBP } & No & 48 & $38.4 \%$ & 77 & $61.6 \%$ & 0 \\
\hline & Yes, but inadequate & 32 & $58.2 \%$ & 23 & $41.8 \%$ & 0 \\
\hline & Yes, adequate & 20 & $43.5 \%$ & 26 & $56.5 \%$ & $0.048^{*}$ \\
\hline
\end{tabular}

Because of missing data, all frequencies do not sum up to the total. Values are frequency/percentage, except if otherwise specified. P90: 90 th centile; ORRAS: operatory room risky activities score.

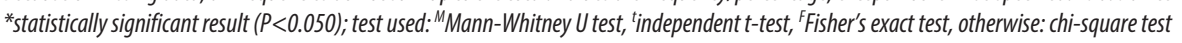


Characterizing Occupational Low Back Pain among Surgeons Working in Ministry of Health Hospitals in Jeddah City... K.S. Aseri et al.

Table 6. Predictors of occupational low back pain among surgeons (multivariate binary logistic regression)

\begin{tabular}{|l|l|l|c|c|c|}
\hline \multicolumn{1}{|c|}{ Predictor } & \multicolumn{1}{c|}{ Level } & OR & \multicolumn{2}{c|}{ P5\% CI } & 0 \\
\hline \multirow{2}{*}{ Exercise (times/week) } & None & Ref & 0 & 1.73 & $0.004^{*}$ \\
\cline { 2 - 6 } & Irregular & 0.73 & 0.31 & 0.61 & 0.470 \\
\cline { 2 - 6 } & Regular & 0.27 & 0.12 & 3.56 & $0.002^{*}$ \\
\hline Other trauma history & Yes & 1.60 & 0.72 & 2.18 & 0.251 \\
\hline Other medical history & Yes & 1.04 & 0.50 & 5.43 & $0.012^{*}$ \\
\hline Other chronic pain & Yes & 2.59 & 1.23 & 1.12 & $0.048^{*}$ \\
\hline ORRAS & Score & 1.06 & 1.00 & 0 & $0.054^{*}$ \\
\hline Education received & No & Ref & 0 & 0.92 & $0.031^{*}$ \\
\cline { 2 - 7 } & Inadequate & 0.41 & 0.19 & 2.89 & 0.669 \\
\cline { 2 - 7 } & Adequate & 1.21 & 0.51 & & \\
\end{tabular}

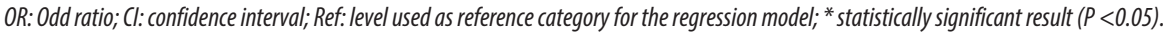

and professional factors including gender, age, marital status, degree, years of experience or years of work, etc. Regarding lifestyle, the prevalence of OLBP was highest among surgeons who practiced no physical exercise $(69.3 \%)$ and decreased to $58.0 \%$ and $41.5 \%$ among those who practiced exercise irregularly and regularly, respectively, and the comparison is statistically significant $(p=0.002)$. Paradoxically, history of back trauma was associated with lower prevalence of OLBP $(33.3 \%$ versus $57.7 \%, P=0.046)$; while history of sport injury $(P=0.081)$, surgery $(p=0.632)$, or other injuries $(P=0.943)$ of the back showed no significant association with OLBP. On the other hand, history of other trauma $(68.6 \%$ versus $52.0 \%, P=0.035)$, other chronic pain $(77.8 \%$ versus $58.3 \%, P=0.006)$, and other medical history $(67.1 \%$ versus $50.6 \%, \mathrm{P}=0.021)$ were significantly associated with a higher prevalence of OLBP, compared to absence of these conditions, respectively.

Analysis of psychological stress showed no difference in the prevalence of OLBP between the different levels of perceived stress at work $(p=0.210)$; however, ORRAS was greater in the group of surgeons with OLBP (mean [SD] $=12.98$ [6.08]) compared to their counterparts (mean [SD] $=11.02[6.43]$ ) and the difference was statistically significant $(p=0.020$ ). Education to prevent LBP was associated with lower prevalence of OLBP compared to no education ( $P=$ 0.048); however, the group of surgeons who declared that the education they received was adequate exhibited relatively higher prevalence $(56.5 \%$ versus $41.8 \%)$ compared to those who declared that the received education was inadequate (Table 5).

\section{Independent Risk Factors of OLBP}

Multivariate regression model including significant factors from the previous analysis showed two protective factors and two risk factors for OLBP. The two independent protective factors were regular physical exercise $(\mathrm{OR}=0.27, \mathrm{P}=0.002)$ and education received that is deemed inadequate by the participant $(\mathrm{OR}=$ $0.41, P=0.031)$. The two independent risk factors were ORRAS score $(\mathrm{OR}=1.06, \mathrm{P}=0.048)$ and the presence of another chronic pain other than the back pain $(\mathrm{OR}=$ 2.59, $\mathrm{P}=0.012$ ) (Table 6).

\section{Discussion}

\section{Summary of Findings}

Performing surgical procedures is a psychologically and physically demanding task, and prolonged discomfort or stress of the surgeons may diminish their operatory skills and compromise their career path. Further, chronic and disabling pain resulting from an occupational disease not only impairs the quality of work life but also affects the overall quality of life of the individual. This study used a double-criterion method to estimate the prevalence of OLBP among surgeons and investigated the associated occupational and extra-occupational factors and predictors. According to the findings, more than one in two (55.8\%) surgeons suffer from OLBP; which was defined as a LBP that first occurred after starting operating room job activity (criterion 1) with high likelihood of being related to job as per the participant's judgment (criterion 2).

\section{Estimation of OLBP: a Methodological Issue}

To date, there is no objective and reliable medical standard to discriminate OLBP from non-occupational LBP; as in many cases occupational and extraoccupational factors interlock or build up. Furthermore, occupational factors may only trigger silent preexisting abnormalities ${ }^{[13]}$. 
In the present study, use of the first criterion aimed at providing a timing-based objective benchmark for OLBP to discriminate it from non-occupational LBP, which reduced the number of cases (prevalence) from $192(85.0 \%)$ to $156(69.0 \%)$. The use of the second criterion, which relied on the participant's appraisal of the relationship between his / her LBP and professional activity, enabled the exclusion of 42 cases with low likelihood of OLBP. Crossing the two criteria aimed at reducing subjectivity in participant's appraisal and lead to excluding 24 cases described as likely being related to occupation, although symptoms onset occurred before starting OR job. Although this method may have some limitations in diagnosis, it could be considered for screening purposes.

\section{Prevalence of OLBP}

In literature, the prevalence of OLBP shows great variation depending on profession and or population, as well as on criteria used to define the outcome. For the present study, the profession and population was surgeons, while the outcome was the work-life prevalence of OLBP. Consistent with the findings, prevalence of LBP among surgeons were remarkably high in literature. For example, in Iran, the point prevalence of LBP among surgeons was reported to be $39.9 \%$, while 1 -year prevalence was $71.7 \%$ and lifetime prevalence was $84.8 \%{ }^{[11]}$; however, the estimates included both occupational and non-occupational, which explains the higher rates compared to the present study. Rates of LBP are also high among operatory room staff similar to the findings from the studies conducted Bin Homaid et al. ${ }^{[10]}$, where LBP was found to be as high as $74.2 \%{ }^{[10]}$, or that in Riyadh, showing $67 \% \%^{[14]}$.

Although these rates are likely to be higher than common LBP in general population, they fall well within the range of estimates reported among healthcare workers, where the prevalence of LBP ranged between $47 \%$ and $74 \% \%^{[13,15,16]}$. Nevertheless, comparison between different specialties points towards a higher risk among surgical positions, as evidenced by a study among healthcare workers in Saudi Aramco that showed a significantly increased risk of back pain among surgeons with a remarkable odds ratio of 5.6 $6^{[17]}$. Similar evidence was reported in a study showing a rate of $65.7 \%$ for LBP among nurses working at a tertiary care hospital in Jeddah; working in surgical departments was associated with the greatest risk $(\mathrm{OR}=2.2)$ of LBP compared to other departments and by reference to medicine ${ }^{[6]}$. This indicates that the operating room working environment comprises specific harm that should be identified and corrected.

\section{Care Seeking Behavior: a Specific Issue}

Assessment of OLBP characteristics showed inadequate care-seeking behavior among surgeons as only one in three of those afflicted have already sought medical advice regarding their OLBP and only one in four have benefited from imaging investigations. This results in a majority of cases (54.8\%) being undiagnosed and probably being inadequately managed and followed up. A meta-analysis showed that health care-seeking behavior among individuals with chronic LBP is mainly determined by the level of disability; while other factors such as being female or having a history of back pain may also be associated with higher rates of care seeking ${ }^{[18]}$. In the present study, cases with disabling LBP are probably excluded from the population and can be considered biased, as only practicing surgeons were included while those on sick leave or unfit for work were not surveyed.

\section{Professional and Psychosocial Impact of OLBP}

Despite the low rates of care seeking among surgeons with OLBP, this study shows evidence of a significant impact on afflicted surgeons' quality of life as up to $25 \%$ of them declared suffering great impact and up to $40 \%$ a moderate impact on various life domains including professional achievement, social relationships, psychological well-being and ADLs. Individuals with chronic LBP generally report poorer perceived health status, decreased quality of life, higher incidence of psychological distress and severe bodily pain than the general population which is frequently associated with constraints in professional and social achievement ${ }^{[19]}$.

\section{To Reduce Physical Stress in Operatory Rooms}

Unhealthy ergonomic conditions are a well-known characteristic of operating rooms, where surgeons are exposed to several tasks involving uncomfortable movements and postures, such as lifting, bending, twisting, etc. This is recognized to increase the risk of musculoskeletal disorders among surgeons as well as other operatory room workers, compared to their peers from other departments ${ }^{[20]}$. The present study examined the association of eight non-ergonomic movements and postures with OLBP among surgeons, using a scoring model based on the frequency of performing each activity. According to this model, a one-unit increment of the overall score (ORRAS) 
is associated with a $6 \%$ odd risk of $L B P(O R=1.06)$. This denotes a probable cumulative effect of nonergonomic activities; besides eventual specific effects of each risky activity, which will be explored further and presented in another paper.

\section{Role of Back Pain Education}

In the present study, having received education to prevent LBP was an independent protective factor against LBP as it reduced the risk by $59 \%$; however, this was only observed among participants who deemed the education as being inadequate. Although this result may seem paradoxical, it may be explained by surgeons who have more frequently experienced LBP being more demanding regarding back education issues. Otherwise, the effectiveness of back education and the relatively recent concept of "back school" in reducing disability and pain among patients with chronic low back pain is thoroughly demonstrated ${ }^{[21]}$. It was further demonstrated that back school programs contribute in improving quality of life and reducing the psychological impact of $\mathrm{LBP}^{[22]}$. From another view, adapting operating rooms by modern and ergonomic standards and equipment may reduce considerably the physical stress ${ }^{[23]}$.

\section{Regular Physical Activity as a Protective Factor for OLBP}

Among the remarkable findings in the present study, regular physical activity being significantly associated with lower prevalence of OLBP was found to be an independent protective factor with approximately $73 \%$ less risk ( $O R=0.27$ ) with reference to the absence of physical activity. On the other hand, irregular exercise was not demonstrated to reduce OLBP in this study. A systematic review and meta-analysis of 36 prospective cohort studies including 158,475 individuals demonstrated that leisure time physical exercise reduced significantly the risk of chronic LBP by $11 \%$ to $16 \%(\mathrm{OR}=0.89$ to 0.84$)$ in a dose-dependent fashion with exercise frequency and intensity ${ }^{[24]}$. Further, moderate to high leisure time exercise constitutes a protective factor against sciatica and lumbar radicular pain, as evidenced by another meta-analysis carried out by the same authors ${ }^{[25]}$. On the other hand, it may be hypothesized that intensive physical activity may have detrimental effects on the back and increase the risk of chronic LBP; however, a prospective Norwegian study including 9,616 women and 8,452 men without LBP at baseline confirmed the beneficial effect of physical activity at all intensity levels without evidencing a U-shaped relationship between physical activity intensity and LBP ${ }^{[26]}$. Additionally, there is extensive evidence supporting the beneficial effect of exercise and physical activity in reducing pain and disability and improving psychological and physical well-being of individuals with chronic back pain, while highlighting the detrimental effects of inactivity in delaying return to normal activity and exacerbating disability and sick leave due to LBP ${ }^{[27]}$. Therefore, many instances, such as the Royal College of General Practitioners (UK), recommended various physical activities and exercise such as aerobic, muscle strengthening, stretching and postural control among patients with nonspecific LBP, with emphasis on structured exercise programs ${ }^{[28]}$. Economically, a randomized controlled trial demonstrated that effective exercise among individuals with nonspecific LBP was associated with a yearly cost savings of approximately US $\$ 3,200$ by patient, compared to normal care, besides a notable gain in quality of life ${ }^{[29]}$. This highlights the importance of promoting physical exercise among the doctors' community and more specifically surgeons. Medical schools and hospitals should endorse regular physical activity among students, residents and practitioners as a preventive measure to reduce the risk of OLBP and other occupational diseases and improve overall physicians' health within a strategic vision to optimize care quality.

\section{Conclusion}

This current study used a double-criterion method to estimate the prevalence of OLBP among Saudi surgeons and found that more than one $(55.8 \%)$ in two surgeons suffered from this condition. Frequent exposure to intraoccupational risky activities is significantly predictive of OLBP, which demonstrates that the operating room working environment comprises specific harm that should be further identified and corrected. Further, chronic and disabling pain resulting from an occupational disease not only impairs the quality of work life but also impacts the overall quality of life of the individual. On the other hand, findings of this study highlight the protective role of regular physical activity as well as the beneficial effect of back pain education, which indicates the relevance of promoting a healthy lifestyle and ergonomic working conditions. 
Characterizing Occupational Low Back Pain among Surgeons Working in Ministry of Health Hospitals in Jeddah City... K.S. Aseri et al.

\section{Conflict of Interest}

The authors have no conflict of interest.

\section{Disclosure}

The authors did not receive any type of commercial support either in forms of compensation or financial for this study. The authors have no financial interest in any of the products or devices, or drugs mentioned in this article.

\section{Ethical Approval}

The study design was reviewed and approved by the Unit of Biomedical Ethics Research Committee at King Abdulaziz University.

\section{References}

[1] Meucci RD, Fassa AG, Faria NM. Prevalence of chronic low back pain: systematic review. Rev Saude Publica 2015; 49.

[2] Ganesan S, Acharya AS, Chauhan R, Acharya S. Prevalence and risk factors for low back pain in 1,355 young adults: a cross-sectional study. Asian Spine J 2017; 11(4): 610-617.

[3] Manchikanti L, Singh V, Falco FJ, Benyamin RM, Hirsch JA. Epidemiology of low back pain in adults. Neuromodulation 2014; 17 Suppl 2: 3-10.

[4] Dagenais S, Tricco AC, Haldeman S. Synthesis of recommendations for the assessment and management of low back pain from recent clinical practice guidelines. Spine J 2010; 10(6): 514-529.

[5] Cougot B, Petit A, Paget C, Roedlich C, Fleury-Bahi G, Fouquet M, Menu P, Dubois C, Geraut C, Roquelaure Y, Tripodi D. Chronic low back pain among French healthcare workers and prognostic factors of return to work (RTW): a non-randomized controlled trial. J Occup Med Toxicol 2015; 10: 40.

[6] Attar SM. Frequency and risk factors of musculoskeletal pain in nurses at a tertiary centre in Jeddah, Saudi Arabia: a cross sectional study. BMC Res Notes 2014; 7: 61.

[7] Leggat PA, Smith DR, Clark MJ. Prevalence and correlates of low back pain among occupational therapy students in Northern Queensland. Can J Occup Ther 2008; 75(1): 3541.

[8] Al Bahrani A, Al Huwaykim M, Al Kuwaiti A, AlAlwi M, Al Dulaim H, Al Mazeedi T, Al Bahrani A, Al Sayafi A, Al Bin Saleh A, Albahrani N, Almohammedali A, Alfayez M, Bin Amir H, Al Hwaykem A, Albahrani T, Bin Amer F. Prevalence of low back pain in healthcare workers in eastern region in Saudi Arabia. Int J Sci Res 2017; 6(1): 1383-1385.

[9] Punnett L, Prüss-Utün A, Nelson DI, Fingerhut MA, Leigh J, Tak S, Phillips S. Estimating the global burden of low back pain attributable to combined occupational exposures. Am J Ind Med 2005; 48(6): 459-469.

[10] Bin Homaid M, Abdelmoety D, Alshareef W, Alghamdi A, Alhozali F, Alfahmi N, Hafiz W, Alzahrani A, Elmorsy S. Prevalence and risk factors of low back pain among operation room staff at a Tertiary Care Center, Makkah, Saudi Arabia: a cross-sectional study. Ann Occup Environ Med 2016; 28: 1.

[11] Mohseni-Bandpei MA, Ahmad-Shirvani M, Golbabaei $N$, Behtash $H$, Shahinfar Z, Fernández-de-las-Peñas C. Prevalence and risk factors associated with low back pain in Iranian surgeons. J Manipulative Physiol Ther 2011; 34(6): 362-70.

[12] Balch CM, Freischlag JA, Shanafelt TD. Stress and burnout among surgeons: understanding and managing the syndrome and avoiding the adverse consequences. Arch Surg 2009; 144(4): 371-376.

[13] Al-Otaibi ST. Prevention of occupational back pain. J Fam Community Med 2015; 22(2): 73-77..

[14] Alobidan AY, Alghamdi KA, Binghali AN, Aldaham M, Alanazi KW, Altamimi. SM. Prevalence of back pain among operation room staff in Riyadh city, Saudi Arabia. Int J Adv Res 2016; 4(12): 159-165.

[15] Mitchell T, O'Sullivan PB, Burnett AF, Straker L, Rudd C. Low back pain characteristics from undergraduate student to working nurse in Australia: a cross-sectional survey. Int J Nurs Stud 2008; 45(11): 1636-1644.

[16] Alnaami I, Awadalla NJ, Alkhairy M, Alburidy S, Alqarni A, Algarni A, Alshehri R, Amrah B, Alasmari M, Mahfouz AA. Prevalence and factors associated with low back pain among health care workers in southwestern Saudi Arabia. BMC Musculoskelet Disord 2019; 20(1): 56.

[17] Behisi MA, Al-Otaibi ST, Beach J. Back pain among health care workers in a Saudi Aramco facility: prevalence and associated factors. Arch Environ Occup Health 2013; 68(1): 30-38.

[18] Ferreira ML, Machado G, Latimer J, Maher C, Ferreira PH, Smeets RJ. Factors defining care-seeking in low back pain-a meta-analysis of population based surveys. Eur J Pain 2010; 14(7): 747.e1-7.

[19] Froud R, Patterson S, Eldridge S, Seale C, Pincus T, Rajendran D, Fossum C, Underwood M. A systematic review and meta-synthesis of the impact of low back pain on people's lives. BMC Musculoskelet Disord 2014; 15: 50.

[20] Vural F, Sutsunbuloglu E. Ergonomics: an important factor in the operating room. J Perioper Pract 2016; 26(7-8): 174178.

[21] Sahin N, Albayrak I, Durmus B, Ugurlu H. Effectiveness of back school for treatment of pain and functional disability in patients with chronic low back pain: a randomized controlled trial. J Rehabil Med 2011; 43(3): 224-229.

[22] Paolucci T, Morone G, losa M, Fusco A, Alcuri R, Matano A, Bureca I, Saraceni VM, Paolucci S. Psychological features 
Characterizing Occupational Low Back Pain among Surgeons Working in Ministry of Health Hospitals in Jeddah City... K.S. Aseri et al.

and outcomes of the Back School treatment in patients with chronic non-specific low back pain. A randomized controlled study. Eur J Phys Rehabil Med 2012; 48(2): 245253.

[23] Klein M, Andersen LP, Alamili M, Gögenur I, Rosenberg J. Psychological and physical stress in surgeons operating in a standard or modern operating room. Surg Laparosc Endosc Percutan Tech 2010; 20(4): 237-242.

[24] Shiri R, Falah-Hassani K. Does leisure time physical activity protect against low back pain? Systematic review and meta-analysis of 36 prospective cohort studies. Br J Sports Med 2017; 51(19): 1410-1418.

[25] Shiri R, Falah-Hassani K, Viikari-Juntura E, Coggon D. Leisuretime physical activity and sciatica: A systematic review and meta-analysis. Eur J Pain 2016; 20(10): 1563-1572.

[26] Heuch I, Heuch I, Hagen K, Zwart JA. Is there a U-shaped relationship between physical activity in leisure time and risk of chronic low back pain? A follow-up in the HUNT Study. BMC Public Health 2016; 16: 306.

[27] Hildebrandt VH, Bongers PM, Dul J, van Dijk FJ, Kemper HC. The relationship between leisure time, physical activities and musculoskeletal symptoms and disability in worker populations. Int Arch Occup Environ Health 2000; 73(8): 507-518.

[28] National Collaborating Centre for Primary Care (UK). Low Back Pain: Early Management of Persistent Non-Specific Low Back Pain [Internet]. London: Royal College of General Practitioners (UK); 2009. (NICE Clinical Guidelines, No. 88.) 6, Physical activity and exercise. Available from: <www.ncbi. nlm.nih.gov/books/NBK11707/\#>.

[29] Hollinghurst S, Sharp D, Ballard K, Barnett J, Beattie A, Evans M, Lewith G, Middleton K, Oxford F, Webley F, Little P. Randomised controlled trial of Alexander technique lessons, exercise, and massage (ATEAM) for chronic and recurrent back pain: economic evaluation. BMJ 2008; 337: a2656. 


\title{
وصف الام أسفل الظهر المهنية بين الجراحين العاملين في مستثفيات وزارة الصحة بجدة :مدى الانتثار، السمات السريرية، المخاطرة المرة وعوامل ملفين الحماية
}

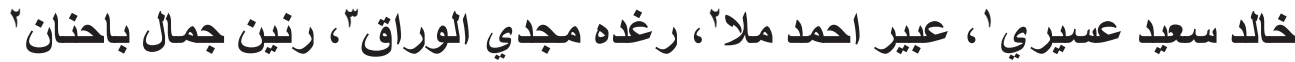

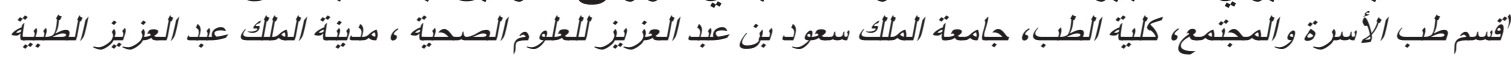 \\ كلبة الطب، كلية ابن سبيا الطبية الطبة كلية

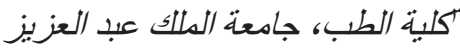 \\ جدة ـ المعلكة العربية السعودية
}

المستخلص. تم إجر اء هذه الدر اسة المقطعية لتحديد نسبة انتشار و عو امل المخاطرة المؤدية إلى آلام أسفل الظهر الناتجة عن فئن

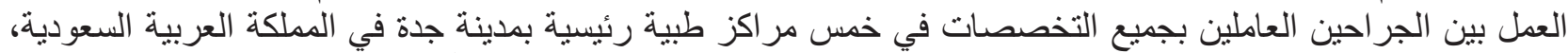

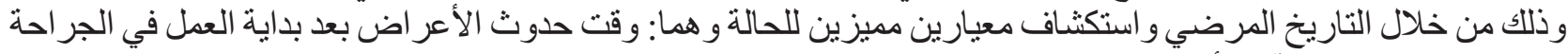

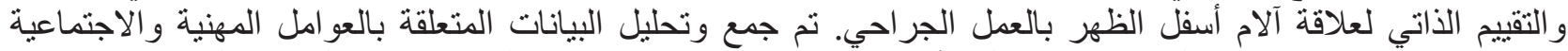

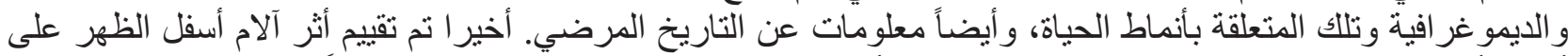

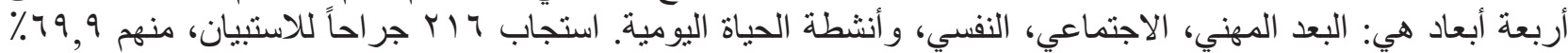

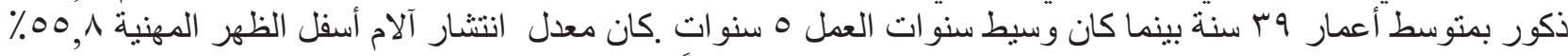

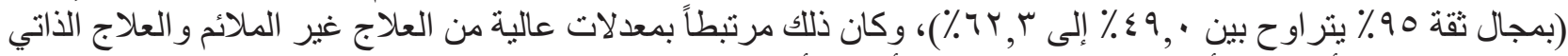

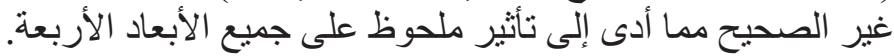

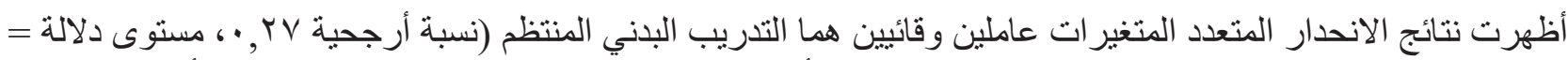

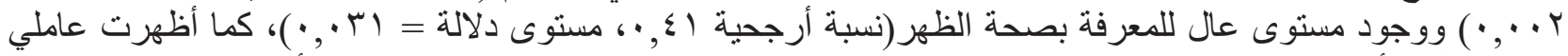

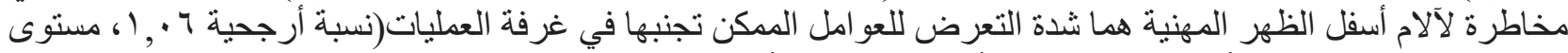

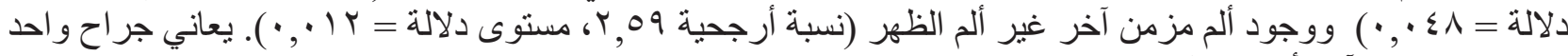

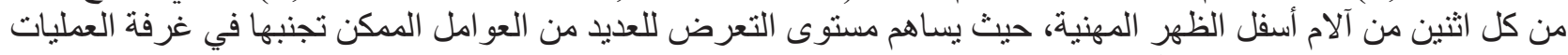
في زيادة خطر التعرض لتلك الآلام. كما برز دور التدريب البدني المنتظم و التنقيف الصحي لصحة الظهر كعاملين وقائيين 